\title{
Killing More than Pain: Etiology and Remedy for an Opioid Crisis
}

\author{
John F. Kelly and Sarah E. Wakeman
}

\section{Abbreviations}

CBT Cognitive-behavioral therapy

CDC Centers for Disease Control and Prevention

EVD Ebola virus disease

FDA Food and Drug Administration

JCAHO The Joint Commission on Accreditation of Healthcare Organizations

MI Motivational interviewing

NA Narcotics Anonymous

OUD Opioid use disorder

PMPs Prescription monitoring programs

SARS Severe acute respiratory syndrome

TSF Twelve-step facilitation

J. F. Kelly $(\bowtie)$

Recovery Research Institute Massachusetts General Hospital, Boston, MA, USA

Center for Addiction Medicine Massachusetts General Hospital, Boston, MA, USA

Elizabeth R. Spallin Professor of Psychiatry in Addiction Medicine, Harvard Medical School, Boston, MA, USA

e-mail: jkelly11@mgh.harvard.edu

S. E. Wakeman

Massachusetts General Hospital Substance Use Disorder Initiative, Boston, MA, USA

Massachusetts General Hospital Addiction Medicine Fellowship, Boston, MA, USA

Department of Medicine, Harvard Medical School, Boston, MA, USA 


\section{Introduction}

The search for effective pain relief has been ever present across human history. The discovery of opium's ability to relieve pain stimulated the refinement of opium and generation of synthetic analogues to create more effective, potent, and faster acting analgesics. The social and economic value of opioid analgesics has remained high not only due to their demonstrated efficiency in mitigating pain but also their noteworthy ability to reliably produce pleasant rewarding psychological side effects. Consequently, at various times during the past several hundred years, opioid drugs have sparked wars, fueled black markets, driven up violence and cartel crime, and triggered numerous opioid addiction and overdose death epidemics around the world.

The therapeutic and humane desire to address pain — one of the most debilitating and disturbing of all human experiences-together with enhanced pharmaceutical discoveries in medication design, manufacture, marketing, sales, and distribution of oral opioid analgesic medications has led more recently to excess availability and accessibility. The additional seductive pharmacological nature of these potent compounds, and the ability to administer doses directly into the bloodstream via hypodermic needles, producing intense euphoria, sedation, and hypnosis (sleep), can lead to some developing compulsive use and addiction. As societies have grappled with rising rates of use and addiction, some have reflexively reached for supplycentric policies to reduce access. While supply reduction is a major piece of the puzzle, without addressing the demand side of the equation, these approaches can have the inadvertent effect of shifting people who were using prescription opioids to the often cheaper, more readily available, but more lethal, options of heroin and illicitly manufactured fentanyl.

In countries such as the United States, between approximately the late 1990s and 2018, rates of population opioid exposure and opioid overdose deaths have risen sharply and steadily creating one of the largest overdose death epidemics in modern history-possibly ever. How we got here, what we can do about it, and how we can prevent it in the future, are the fundamental questions that we attempt to answer in this chapter. To begin, below we describe briefly the nature, structure, origin, and growth in the use of opioid analgesics and subsequently describe the etiology and epidemiology of the current crisis using broad public health and health belief models as frameworks to help guide understanding. In the second section, we describe the approaches that have been applied to address both supply (e.g., overprescribing) and demand (e.g., overdose intervention, pharmacological and psychosocial treatments) sides of the equation. In the final section, we review the lessons learned in an attempt to outline strategies to prevent future epidemics.

\section{Context: Origin and Growth in the Use of Opioids}

\section{Poppies, Pain, and Panacea}

A large variety of natural, semisynthetic, and fully synthetic opioid-based medications have become increasingly available as the extraction, synthesis, and 
manufacturing processes of opioids have become more efficient and refined. The opium poppy, from which the morphine molecule comes, is a naturally occurring flowering plant found in many parts of the world and cultivated explicitly in China and parts of the Middle East for its pain killing and euphoria-inducing properties for both licit and illicit markets. Opium is the dried aqueous gum that can be leached from the opium poppy, about $12 \%$ of which is the alkaloid analgesic, morphine. Morphine can be extracted, and a more potent formulation can be derived (diacetylmorphine or heroin), which is used as a strong analgesic to address severe pain as well as used recreationally to induce rapid and intense euphoria.

The discovery and use of morphine from the opium poppy is recorded dating back to ancient Egypt. Morphine and its derivatives (codeine) can also be used effectively to treat a range of other common ailments including severe cough and diarrhea, but opioids' refinement and widespread synthetic analogue manufacture (e.g., fentanyl) is a product of modern biochemistry and the pharmaceutical industry.

Pharmacodynamically, opioids are broken down upon entering the body and bind to the mu-opioid receptor producing analgesia, euphoria, and anxiety-reducing effects. In high enough doses, however, these compounds can produce respiratory depression and death. When mixed with other sedative-hypnotic drugs, such as alcohol or benzodiazepines, respiratory depression is exacerbated and the risk of fatal overdose increases. Research suggests that for each additional illicit drug administered in combination with an opioid, the risk of death from opioids doubles [1], and, in fact, many fatal overdoses are caused in this synergistic fashion.

\section{Killing More Than Pain: The Double-Edged Sword of Opioids}

The discovery of the pain-killing potential of opium-based tinctures and, later, more potent pharmaceutically enhanced analogues has been both an immense blessing and at the same time a worrisome curse - "too much of a good thing" can create problems. The degree of use of pharmaceuticals, like many other commodities, is socially, economically, politically, and culturally based. The United States is among the largest global consumers of opioids, with prescribing rates that outpace many peer nations; however, while consumption of opioids in the United States is undoubtedly high, it has been overstated. For example, while it is commonly reported that the United States consumes " $80 \%$ of the world's supply of opioids," it is because within that figure are certain opioid medications that are exclusively marketed in the United States while other countries use similar, but slightly different, opioid compounds. Consequently, a more accurate estimate is that while the United States has approximately $4.4 \%$ of the world's population, it consumes about $30 \%$ of the world's global supply of opioids [2]. Still, as shown in Fig. 1.1, using standard daily doses per million inhabitants, relative to other middle- and high-income countries, opioid use is disproportionately high but not as high as is commonly stated. Perhaps the interplay of a strong biotech industry, capitalist consumer-oriented free market economy, and constitutionally based cultural expectations that explicitly endorse the right to "the pursuit of happiness" has promoted America's demand for rapid pharmaceutical remedies for pain and suffering. Within such an economic and 
sociocultural context, however, like many industrialized countries, the United States has sought to institute safeguards to curb the potential appetite for "too much of a good thing." For example, it possesses among the strongest protections and regulations worldwide regarding quality and safety of medications, and administrative oversight regarding scheduling, prescribing, and marketing (e.g., the Food and Drug Administration [FDA]; Prescription Monitoring Programs [PMPs]). As explained below, a number of forces converged to create the current overdose epidemic and opioid addiction crisis seen that has emerged since the late 1990s.

\section{Conceptual Models for Understanding Drug Epidemics}

It can be helpful to draw upon well-developed conceptual frameworks for helping to understand, and thus intervene upon, different disease epidemics that occur from time to time. The public health model of disease suggests there are three major factors that interact to influence the spread of an epidemic: the agent (e.g., the presence of an environmental toxin; in this case, opioids), the host (i.e., the human beings exposed to the opioid), and the environment (e.g., the variables that can influence the degree to which the host is exposed to the opioid; see Fig. 1.1). To the extent that an agent (e.g., opioid) can cause harm to a host (person), the environment can play a crucial role in mitigating or exacerbating that harm. In the case of addiction or substance use disorder, more generally, this public health model of disease is complicated slightly further, because — as is the case with opioids - the agent is highly desired by the host since it can kill pain as well as produce intense pleasure. All other things being equal, if it becomes widely available, easily accessible, affordable, legal, and medically prescribed-and thus has lower stigma attached to it-its use will increase. Given that opioids are known to cause

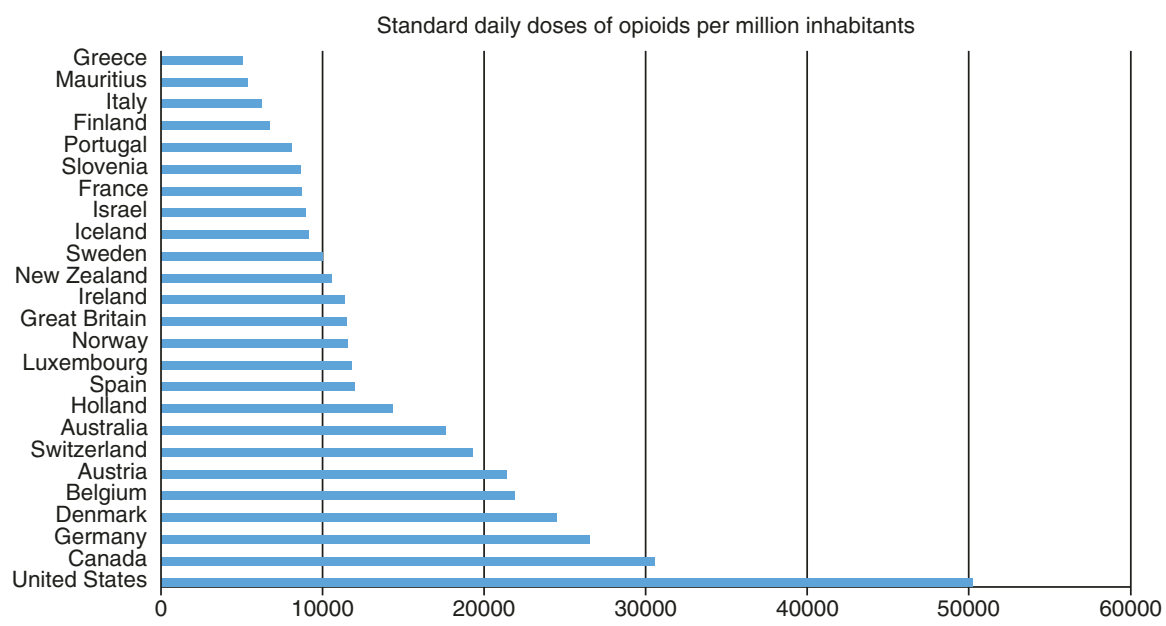

Fig. 1.1 Standard daily doses of opioids per million inhabitants across developed nations [2] 
addiction and increase risk for overdose death and are heavily regulated for safety and quality, how could these potent and seductive medications become so widely available and easily accessible?

It seems likely that two major factors influenced this high degree of prescription use of opioids and population exposure. One was the perception that these medications are very safe and hardly ever led to addiction among pain patients. Strikingly, based upon a single, one-paragraph publication in 1980 [3] —albeit one that was published in perhaps the most highly influential medical journal of all (the New England Journal of Medicine) — that was heavily promoted and cited, it was widely believed that addiction was almost nonexistent among medical patients treated in hospital settings with opioid analgesics such as hydrocodone and hydromorphone. Given the journal's medical prestige, this publication and its common citation were rarely questioned and became widely referred to with little scrutiny. The pharmaceutical industry was only too happy to take advantage of what appeared to be a scientific medical blessing on the liberal use of their opioid analgesics from which they stood to profit.

A perceived strong scientific and medical sanction regarding safety and efficacy opened the door to advertising to physicians as well as directly to consumers. The Health Belief Model [4] suggests that people are likely to take a health-related behavioral preventative action if they perceive that the action will decrease the chances of a particular personal negative health outcome. If people believe that the threat is low or practically nonexistent, or not severe, then they are unlikely to engage in such a preventive action. In this case, consumers were led to believe that there was no threat and encouraged to take such medications by health-care providers-culturally, highly trusted entities, who similarly believed these medications had very low risk for harm.

At the same time in the United States, there was a push from several organizations to address pain much more aggressively and there was widespread belief that pain was being inadequately assessed and poorly treated in medical settings. Unrelieved pain was considered to be a major, yet completely avoidable, public health problem. In 1997, a collaborative project was initiated to integrate pain assessment and management into clinical best practice standards. Furthermore, in 2001, all patient care organizations accredited by the Joint Commission on Accreditation of Healthcare Organizations (JCAHO) were required to document proper management of pain, and pain became the "fifth vital sign" — as integral to standard basic medical "best practice" assessment as obtaining a patient's body temperature or blood pressure reading. Adding to this is the fact that prescription opioids were purported and perceived to be "safe" and "nonaddicting," and there was a perfect storm brewing which since then has created hundreds of thousands of casualties.

It was not just the number of prescriptions that went up exponentially during the 1990s and 2000s but also the amount of opioid pills allotted per prescription. Often dozens of potent opioid pills were prescribed for fairly minor to moderate and shortlived, acute pain (e.g., tooth extraction, minor surgery), such that only a fraction of the prescribed pills were needed creating an accumulating glut of residual unused prescription opioids, unwittingly increasing availability and potential accessibility in the community. 
For any desirable commodity, availability, accessibility, and price are major factors influencing a product's consumption. Other things being equal, the more available, accessible, and cheaper a desirable product is, the greater is its consumption. When asked in national surveys conducted in the United States from where individuals had obtained their (prescription) opioids, 54\% reported that they obtained them from a friend or relative from a medicine cabinet at home or from a friend or relative who obtained them from their own medicine cabinet [5]. While direct prescription to individual pain patients puts some at risk of developing addiction, likely to be an even larger issue was how these excess pills flooded the community, increasing access and nonmedical use.

Combining the conceptual frameworks of the public health model and the Health Belief Model, Americans ("host") perceived a decrease in risk and an increase in perceived safety, while opioids ("agent") became more available and accessible, and the cultural, political, and social environment was such that it was completely legal and medically sanctioned to be using these powerful medications without concern (Fig. 1.2). Price too was relatively low in the early years of the epidemic, because for the most part prescribed opioids were covered by insurance, or otherwise cheap. This fact tended also to bias in favor of White American consumers who more likely had health insurance, who were potentially more likely to be prescribed due to unconscious racial bias among physicians, and who, in contrast to prior opioid epidemics, became overrepresented among those overdosing on opioids. Compared to prior mostly male, urban-based, heroin epidemics of the 1960s where the average age of onset was around 16 years old, individuals misusing opioids between 2000 and 2014 were older (mean age, 22.9 years) men and women living in less urban areas who were introduced to opioids through prescription drugs (75.0\%). Also, Whites and non-Whites were equally represented in those initiating use prior to the 1980s, but nearly $90 \%$ of those who began use between 2000 and 2014 were White [6]. In more recent years, however, Blacks and Hispanic and Latino Americans have shown steady increases in overdose deaths, and overdose deaths among Black Americans in particular have risen more sharply since 2014 accounted for largely by an increase in overdoses among older Black men (Fig. 1.3. CDC, 2018).

Fig. 1.2 Public health model framework for understanding drug epidemics

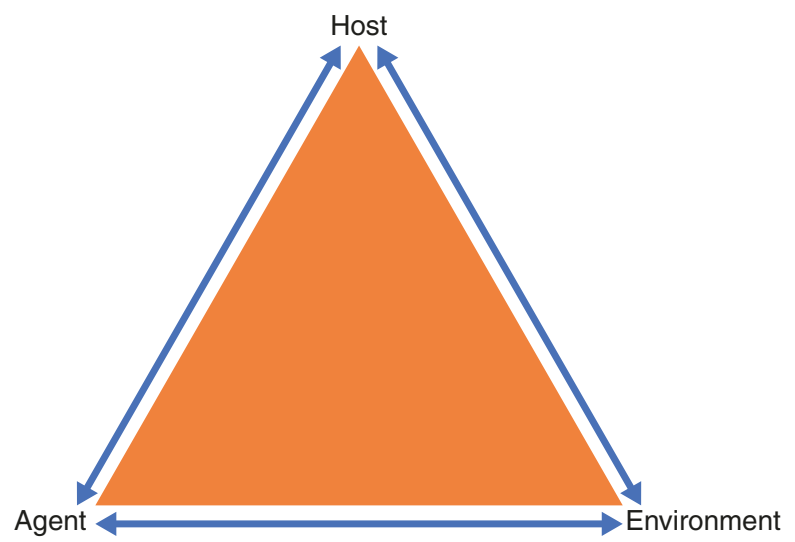


Age-adjusted death rate $\neq$ for drug poisoning by race and hispanic origin, all ages, both sexes: united states, 1999-2016

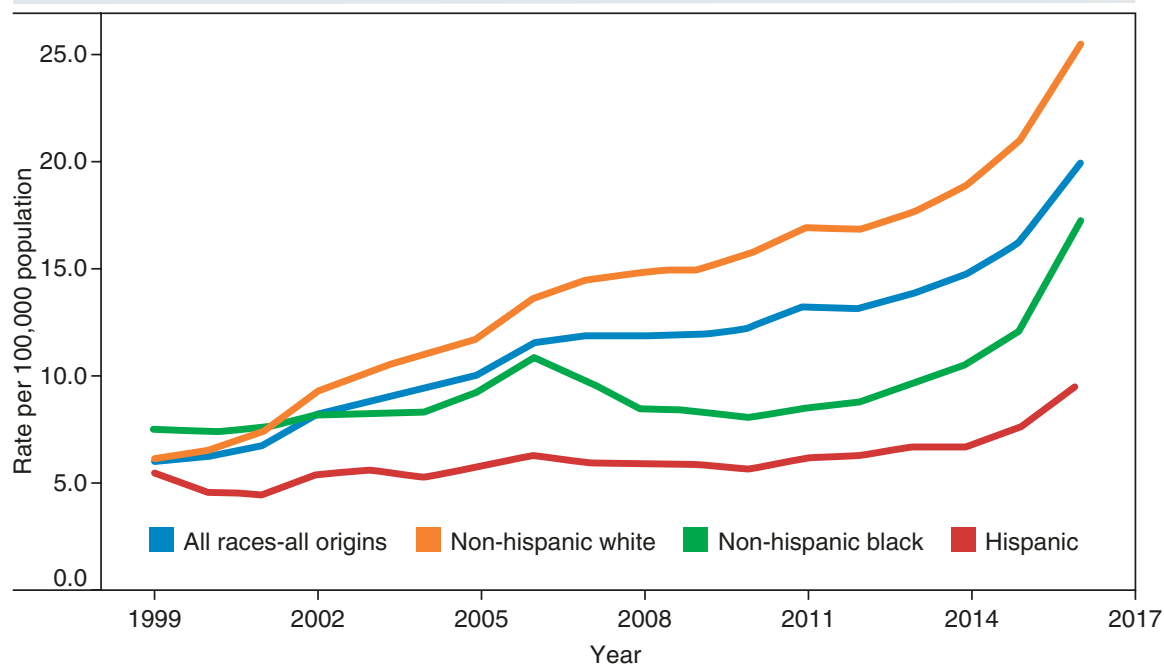

Age-specific death rate $\neq$ for drug poisoning by race and hispanic origin, 55-64, both sexes: united states, 1999-2016

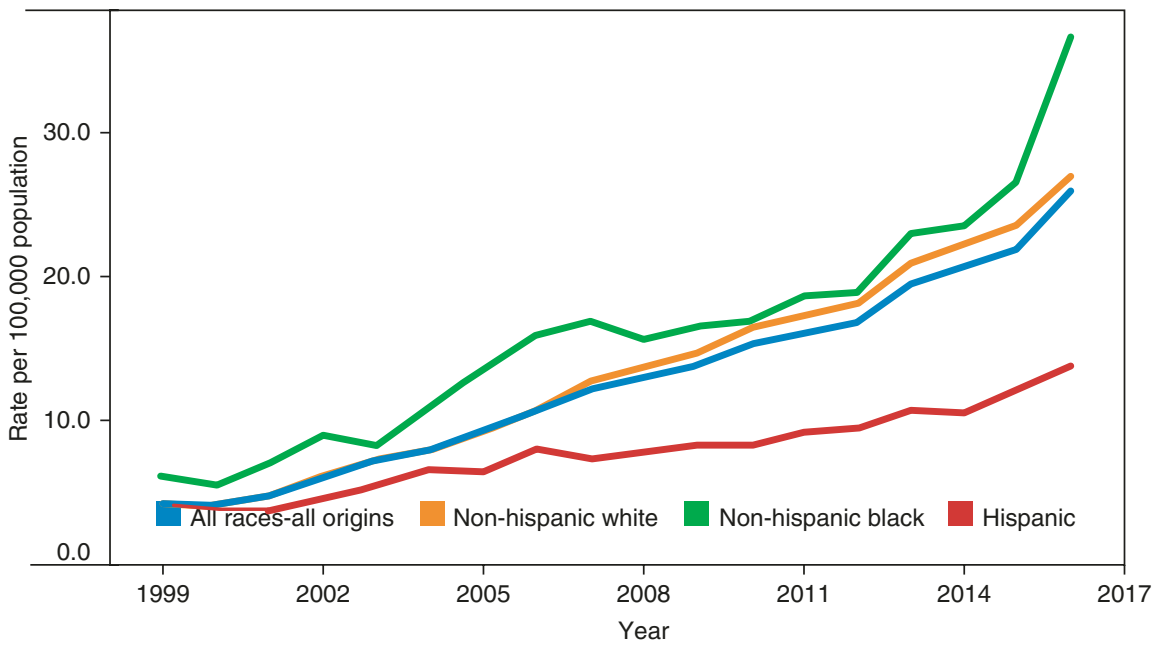

Fig. 1.3 Changes in opioid overdose death rates by racial-ethnic groups. (From CDC. Centers of Disease Control. Drug Poisoning Mortality in the United States, 1999-2016. Accessed at: https:// www.cdc.gov/nchs/data-visualization/drug-poisoning-mortality/)

\section{Epidemiology of Opioid Misuse, Opioid Use Disorder, and Overdose Deaths}

For the reasons outlined above, between 1999 and 2016 there has been a large increase in the numbers of people misusing prescription opioids in the United States, 
in those meeting criteria for an opioid use disorder, and in overdose deaths. Strikingly, overdose is now the leading cause of death for people under the age of 50 in the United States [7]. From 1999 to 2016, for example, more than 630,000 people have died from a drug overdose and it estimated that approximately twothirds of these involved opioids specifically.

According to the US Centers for Disease Control and Prevention (CDC), in 2016, the number of overdose deaths involving opioids (including prescription opioids and illegal opioids like heroin and illicitly manufactured fentanyl) was five times higher than that in 1999 [8]. Currently, on average, 115 Americans die every day from an opioid overdose. In 2016, approximately 42,000 of the more than 64,000 individuals who died from an overdose died from an opioid overdose (Fig. 1.4). In addition, 11.5 million Americans misused prescription opioids and 2.1 million met criteria for an opioid use disorder in 2016. These rates may underestimate the true prevalence of opioid use disorder as they are based on household data which excludes institutionalized, homeless, or incarcerated individuals. A recent study in Massachusetts found the prevalence of opioid use disorder to be $4.6 \%$ [9].

In the current opioid crisis that has resulted in so many opioid overdose deaths, there have been three broad waves (Fig. 1.5). The first large increase began in 1999 with a steady linear rise in deaths from prescription opioids. This was followed by a second wave of heroin-related overdose deaths beginning in 2010, followed by a third wave in synthetic overdose deaths beginning in 2013. Opioid misuse and opioid use disorders are more common among men than women, but both have experienced increasing overdose death rates at roughly the same increased rate since 1999.
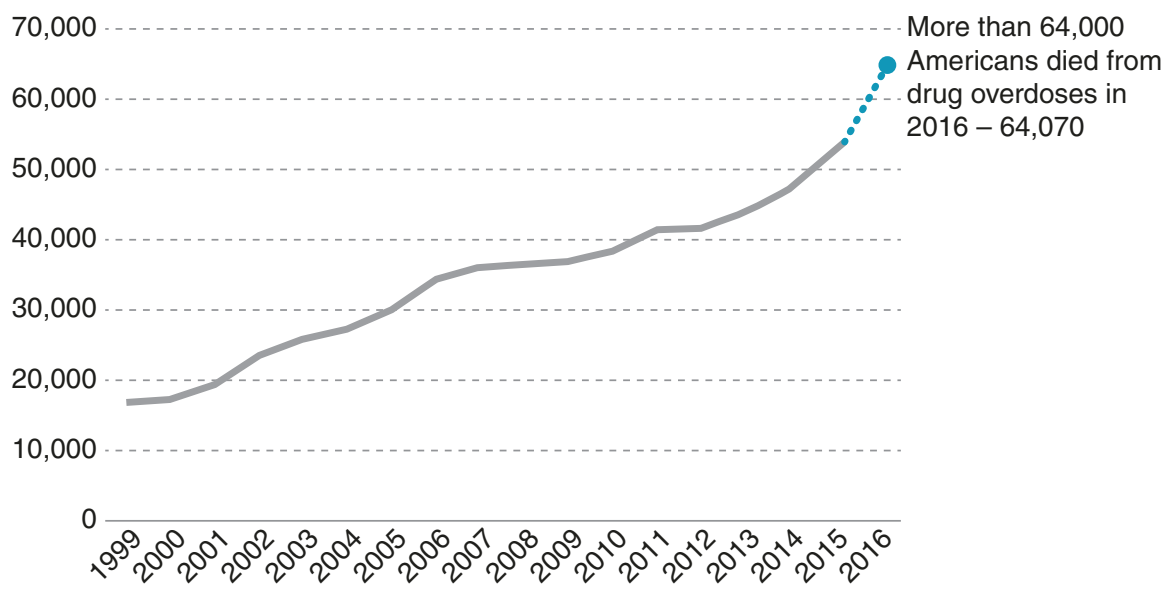

Fig. 1.4 Increasing trend in US overdose deaths 1999-2016. Total US drug deaths. (Source: CDC WONDER. Accessed at: https://wonder.cdc.gov/) 


\section{3 waves of the rise in opioid overdose deaths}

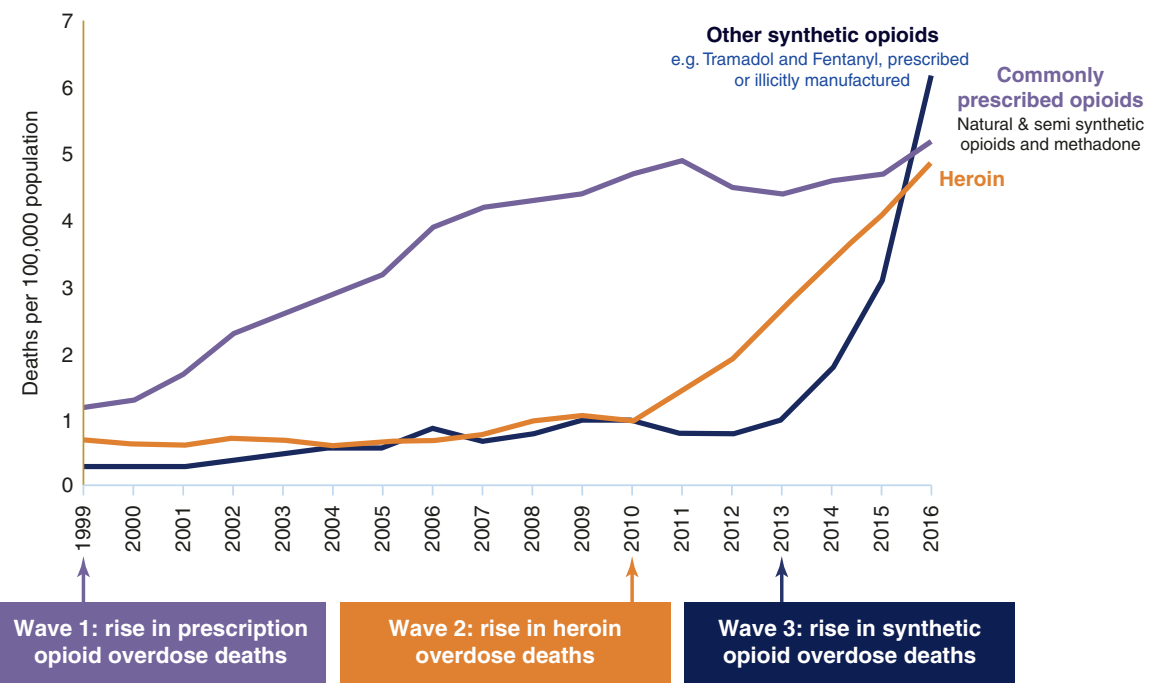

Fig. 1.5 Trends in opioid overdose death rates in relation to prescription opioids, heroin, and synthetic opioids (e.g., fentanyl). (Source: CDC 2018 https://www.cdc.gov/drugoverdose/epidemic/index.html - Response to the Crisis: Reducing Supply, Increasing Prevention, Treatment and Recovery Supports. National Vital Statistics System Mortality File)

\section{Addressing Opioid Misuse, Disorders, and Overdose Deaths}

Considering the public health model once again of agent (opioids), host (people), and environment, there have been increasing concerted attempts to reduce the supply of opioids (the agent) into the environment through prescriber education, prescribing limits, and prescription monitoring program initiatives. There have also been large-scale efforts to protect the host from fatal overdose through education and prevention efforts, as well as emergency intervention efforts (e.g., with widespread distribution of intranasal naloxone [Narcan]) and increased access to medications, psychosocial interventions, and harm reduction strategies (safe injection facilities and syringe service programs). From a policy standpoint, the latter are collectively known as "demand reduction" efforts in the supply and demand equation as they are designed to reduce the appeal of, and desire for, opioids through treating opioid use disorders.

\section{Reducing Supply and Accessibility to Opioids}

As noted, given that availability and accessibility are two major contributors to increased consumption of opioids, there has been a major concerted effort to change prescribing practices through education, monitoring, and restrictions, as well as "take 
back" programs designed to reduce exposures in the general population. States and insurers have begun passing legislation and implementing policies aimed at curtailing access to prescription opioids. While responsible and cautious prescribing of opioids is critically important to reduce unnecessary exposure and access for opioid-naïve individuals to reduce the incidence of opioid use and use disorder, supply-focused strategies are unlikely to benefit people who are already using opioids, including those with opioid use disorder or chronic pain. Prescribing guidelines, such as the 2016 CDC guidelines [10], offered approaches for more judicious use of opioids limiting exposure to opioids in acute pain, continuously addressing the risk and benefit of opioid treatment, and screening and offering treatment for opioid use disorder. At the same time, these guidelines recognized the complexity of managing individuals who were started on high doses of opioids previously and the importance of safeguarding against patient abandonment in these scenarios in the case of opioid use disorder (Fig. 1.6).

CDC recommendations for prescribing opioids for chronic pain outside of active cancer, palliative, and end-of-life care.

Determining When to Initiate or Continue Opioids for Chronic Pain

1. Nonpharamacologic therapy and nonopioid pharmacologic therapy are preferred for chronic pain. Clinicians should consider opioid therapy only if expected benefit for both pain and function are anticipated to outweight risks to the patient. If opioids are use, they should be combined with nonpharmacologic therapy and nonopioid pharmacologic therapy, as appropriate.

2. Before starting opioid therapy for chronic pain, clinicians should establish treatment goals with all patients, including realistic goals for pain and function, and should consider how therapy will be discontinued if benefits do not outweigh risks. Clinicians should continue opioid therapy only if there is clinically meaningful improvement in pain and function that outweighs risks to patient safety.

3. Before starting and periodically during opioid therapy, clinicians should discuss with patients known risks and realistic benefits of opioid therapy and patient and clinician responsibilities for managing therapy.

Opioid Selection, Dosage, Duration, Follow-Up, and Discontinuation

4. When starting opioid therapy for chronic pain, clinicians should prescribe immediate-release opioids instead of extended-release/long-acting (ER/LA) opioids.

5. When opioids are started, clinicians should prescribe the lowest effective dosage. Clinicians should use caution when prescribing opioids at any dosage, should carefully reassess evidence of individual benefits and risks when increasing dosage to $\geq 50$ morphine milligram equivalents (MME)/day, and should avoid increasing dosage to $\geq 90 \mathrm{MME} /$ day or carefully justify a decision to titrate dosage to $\geq 90 \mathrm{MME} /$ day

6. Long-term opioid use often begins with treatment of acute pain. When opioids are used for acute pain, clinicians should prescribe the lowest effective dose of immediate-release opioids and should prescribe no greater quantity than needed for the expected duration of pain severe enough to require opioids. Three days or less will often be sufficient; more than seven days will rarely be needed.

${ }^{\star}$ All recommendations are category A (apply to all patients outside of active cancer treatment, palliative care, and end-of-life) except recommendation 10 (designated category $\mathrm{B}$, with individual decision making required); see full guideline for evidence ratings.

Fig. 1.6 US Centers for Disease Control and Prevention Opioid Prescribing Guidelines. (Source: MMWR. Morbidity and Mortality Weekly Report. March 15, 2016. US Department of Health and Human Services/Centers for Disease Control and Prevention)
7. Clinicians should evaluate benefits and harms with patients within 1 to 4 weeks of starting opioid therapy for chronic pain or of dose escalation. Clinicians should evaluate benefits and harms of continued therapy with patients every 3 months or more frequently. If benefits do not outweigh harms of continued opioid therapy, clinicians should optimize other therapies and work with patients to taper opioids to lower dosages or to

Assessing Risk and Addressing Harms of Opioid use

8. Before starting and periodically during continuation of opioid therapy, clinicians should evaluate risk factors for opioid-related harms. Clinicians should incorporate into the management plan strategies to mitigate risk, including considering offering naloxone when factors that increase risk for opioid overdose, such as history of overdose, history of substance use disorder, higher opioid dosages ( $\geq 50 \mathrm{MME} /$ day), or concurrent benzodiazepine use, are present.

9. Clinicians should review the patient's history of controlled substance prescriptions using state prescription drug monitoring program (PDMP) data to determine whether the patient is receiving opioid dosages or dangerous combinations that put him or her at high risk for overdose. Clinicians should review PDMP data when starting opioid therapy for chronic pain and periodically during opioid therapy for chronic pain, ranging from every prescription to every 3 months.

10. When prescribing opioids for chronic pain, clinicians should use urine drug testing before starting opioid therapy and consider urine drug testing at least annually to assess for prescribed medications as well as other controlled prescription drugs and illicit drugs.

11. Clinicians should avoid prescribing opioid pain medication and benzodiazepines concurrently whenever possible.

12. Clinicians should offer or arrange evidence-based treatment (usually medication-assisted treatment with buprenorphine or methadone in combination with behavioral therapies) for patients with opioid use disorder. taper and discontinue opioids. 
Unfortunately, the application of these guidelines in some cases has been much less nuanced, with some states and insurance companies moving to enact absolute limits on prescription opioid use. These sorts of black-and-white policies risk harming people who have been stably treated on chronic opioid therapy. Dramatic reductions in access to prescription opioids also risk pushing people with opioid use disorder toward the use of heroin or other illicit opioids obtained "on the street." The 2017 report by the National Academies of Science, Engineering, and Medicine acknowledged this potential negative impact of supply-focused policies through "squeezing the balloon" where efforts to reduce the misuse of prescription opioids may actually increase other use of potentially even more dangerous opioids, such as heroin [11]. An example of this was the reformulation of OxyContin into a deterrent formulation intended to prevent misuse, which was associated with a transition to heroin use and an increase in heroin-related overdose deaths.

A further strategy to reduce exposure to prescription opioids was to reduce accessibility via community-based "take back" programs. These initiatives were designed so that individuals could drop off unused opioid medication at local police stations or increasingly at pharmacy "drop boxes" at no cost and no questions asked.

\section{Demand Reduction: Prevention, Treatment, and Recovery Support Services}

A number of evidence-based medications, psychosocial interventions, and recovery support services are available to help people suffering from opioid use disorders. Particularly effective in treating opioid use disorder are the agonist therapies methadone and buprenorphine, with the latter being combined with naloxone to create a therapeutic hybrid buprenorphine/naloxone marketed as "Suboxone." The most scientifically rigorous systematic quantitative reviews of placebo-controlled randomized trials indicate that buprenorphine is an effective medication in the maintenance treatment of heroin addiction, retaining people in treatment at any dose above $2 \mathrm{mg}$ and suppressing illicit opioid use (at doses $16 \mathrm{mg}$ or more) based on placebocontrolled trials. However, compared to methadone, buprenorphine retains fewer people when doses are flexibly delivered and at low fixed doses. If fixed, medium or high, doses are used, buprenorphine and methadone appear no different in effectiveness (retention in treatment and suppression of illicit opioid use) [12]. For prescription opioid addiction, similar results have been found (although the quality of evidence is not as good as it is for heroin addiction). Another rigorous systematic review of clinical trials, for example, found little to no difference between how well methadone and buprenorphine worked to keep people in treatment, to reduce opioid use, or in the side effect profile. The conclusion was that buprenorphine keeps more people in treatment, reduces opioid use, and has fewer side effects compared to detoxification or psychological treatment alone [13].

Antagonists, such as naltrexone, which block the mu-opioid receptor (instead of agonizing it like buprenorphine or methadone), thus preventing the reinforcing effects from opioids should they be used, have been tested among individuals with opioid use disorder. A systematic review of oral naltrexone found it be no better than 
placebo or detoxification [14], presumably due to the lack of compliance with oral/ daily administration. Once per month injectable extended-release naltrexone, in contrast, fares better, especially when it is administrated following initial medically supervised withdrawal to achieve seven to ten days of opioid abstinence. In intentto-treat analyses, buprenorphine is more effective as a treatment for opioid use disorder; however, among the subset of patients who are able to complete medically supervised withdrawal, extended-release naltrexone has similar efficacy to buprenorphine in preventing relapse to illicit opioids $[15,16]$.

Consequently, in sum, there is overall generally good quality, coherent, and consistent experimental evidence supporting agonist, and to a lesser degree, antagonist, medication treatment for opioid use disorder. These are among the most effective treatments for any substance use disorder and should be considered first-line approaches in addressing opioid addiction.

Detoxification, stabilization, and psychological treatments (e.g., cognitivebehavioral therapy [CBT], twelve-step facilitation [TSF], and motivational interviewing [MI]-based interventions) without medications tend not to perform as well, and while popular, little is known about the efficacy of mutual-help organizations, such as SMART Recovery or Narcotics Anonymous, in facilitating and aiding OUD remission [17]. Adding specialized addiction counseling (e.g., CBT for addiction) to agonist medication, therapies that already come with brief 20-45-minute prescriber counseling and checkup - a manualized intervention known as "medical management" (MM) — have not been shown to enhance outcomes among OUD patients [18]. The failure to show additional benefit for specific addiction counseling on top of MM and medications (e.g., buprenorphine/naloxone) may be because MM is likely to mobilize the same kinds of therapeutic mechanisms (e.g., recovery motivation, active coping, increased recovery self-efficacy) that are mobilized by all active interventions $[19,20]$ and, thus, adding an additional specific intervention does not confer additional benefit. Similar kinds of null effects have been found in other studies where psychosocial interventions are increased in intensity (e.g., from 5 hours of therapeutic contact to 20 hours) but do not confer increased therapeutic benefit [21]. There is some preliminary evidence for potential therapeutic synergy from extended community-based interventions, such as Narcotics Anonymous (NA) participation in addition to buprenorphine or methadone [22]. At least one large observational study found that opioid use disorder patients on buprenorphine/naloxone (Suboxone), who engaged more in NA, had significantly better retention on the medication and higher abstinence rates [23]. More studies are needed in these areas to help determine who in particular may benefit from these additional community services to aid long-term remission and recovery.

A large number of individuals suffering from OUD come into contact with the treatment system via the criminal justice system. There has been a growth in so-called drug courts which have been increasingly used to help individuals with opioid use disorder access treatment rather than jail or prison [24, 25]. Compared to adjudication as usual or jail/prison time, drug courts provide access to opioid use disorder treatment and use a combination of monitoring and oversight in treatment to help offenders initiate remission and gain a foothold in 
recovery. Evidence on these entities specifically are somewhat mixed [24, 25], but the quality of evidence is low.

There is some evidence that mandated treatment via the criminal justice system produces outcomes as good as or better than more "voluntary" patients. Rather than suggesting that incarceration or mandated treatment is superior to increased accessibility of voluntary treatment, these better outcomes may be because the criminal justice system facilitates treatment exposure earlier than would normally happen if individuals are left to their own devices, and such mandated patients tend, thus, to have better prognoses [26] and shorter time to remission [27, 28]. In general, the criminal justice system can play a powerful role in truly diverting individuals with OUD into treatment settings rather than detention, incarceration, or correctional supervision such that they begin the treatment process earlier.

Recent recognition of the intransigence of the current opioid crisis by law enforcement officers has led to innovative efforts by some police departments (e.g., Gloucester, MA) to facilitate access to treatment rather than criminal prosecution for individuals with addiction who present themselves at the police station. In Burlington, VT, a partnership between the police department, the mayor's office, the hospital, and the state attorney has sought to increase access to medication treatment for opioid use disorder including not prosecuting people for buprenorphine diversion. This type of approach from a police department and state attorney represents a massive philosophical shift and a recognition by law enforcement that lower threshold access to care, rather than punishment, is the key to addressing the ongoing crisis. These types of approaches to be spontaneously initiated by law enforcement reflect a recognition and increased appreciation of potentially ineffective, traditional, law enforcement approaches to addressing opioid use disorder.

From the standpoint of preventing morbidity and mortality for those with opioid use disorder, demand-focused interventions are by far the most effective. Treatment with medications such as buprenorphine and methadone has been shown to reduce mortality risk by between $50 \%$ and $80 \%$ [29]. Engagement in treatment and ongoing recovery supports reduces the likelihood of ongoing opioid use and its associated harms [22, 30, 31]. Lowering the barriers to treatment access through integrating addiction care into the medical system, ensuring insurance parity for services, and restructuring care models to focus on engagement and retention increase the likelihood that individuals with opioid use disorder will get care and reduce or stop ongoing opioid use [30].

Importantly, given the high degree of heterogeneity in clinical histories and presentations, and clinical course of opioid and other substance use disorders, it is often stated that "one size does not fit all." Many patients, for example, will not take medications despite their proven efficacy. For these patients, proven alternatives should be on the menu of additional options so that patients can choose another option that could help the patient engage and improve their quality of life. These may take the form of long-term residential options such as recovery housing [32], which have shown to be helpful and cost-effective along with the use of other recovery supports such as recovery community [33]. Such centers can assist in helping people get jobs and get connected to other recovery support services (e.g., recovery coaching) that can help sufferers build recovery capital and instill hope for the future. 


\section{Harm Reduction Strategies}

Not all those with opioid use disorders are able or want to stop using opioids for a variety of reasons. For such individuals, a variety of services and strategies have been developed and increasingly deployed to positive effect in many countries around the world. These are designed to reduce overdose deaths as well as lower the potential for transmission of infectious diseases, such as HIV and hepatitis C. Quantitative reviews including meta-analyses support the implementation of such approaches $[34,35]$. In the United States these have taken the form of syringe service programs, overdose education and naloxone ("Narcan") distribution programs, and lower threshold treatment models. Other countries have expanded harm reduction even further to offer supervised injection facilities and supervised prescription heroin and injection programs (e.g., in the United Kingdom; [31]). While cities in the United States have begun exploring the possibility of opening supervised injection facilities, strong opposition from the federal government remains. Evidence mostly from studies conducted in two cities, Vancouver, Canada, and Sydney, Australia, supports the public health utility of safe injection facilities [36], but findings are somewhat mixed in high-quality studies in this domain. That said, there is no evidence that these facilities increase rates of drug use in any capacity. More research, however, is needed to understand the clinical and public health benefits related to these facilities and who in particular is likely to benefit from them.

An initial wave of rising prescription opioid use and related overdose deaths has since been surpassed by a second wave of heroin use and overdose and then illicitly manufactured fentanyl and related death. Addressing the crisis requires an understanding and application of public health models which focus not only on supply reduction but importantly also on addressing demand through treatment and harm reduction.

\section{Conclusions and Future Directions}

Most middle- and high-income countries globally have become largely inured to the endemic premature mortalities related to more commonly used substances such as alcohol and tobacco. While these account for a much larger number of deaths and economic and social harms than opioids each year, the devastation wreaked by these substances, their casualties, and the associated blood and tears are all relatively willingly absorbed into the social fabric. These are not news. What is news is the rapid rise and spread of new substance-induced casualties and the tragic premature end to so many additional lives as a result of opioid overdose. While much needs to be done to address all substance use disorders, the novelty and surge of this particular epidemic begs the question as to how such new tragedies can be prevented in future. What can be learned from this crisis and put to good use for the benefit of future generations?

To begin, there was a clear disconnect between clinical practice and adequate science on safety and addiction potential regarding new prescription opioids. There 
has been an often-cited gap between practice and science for addiction treatment services, whereby practitioners either are not knowledgeable about or otherwise fail to implement science-based "best practices." In this instance, however, it was a slightly different take on this criticism. Providers believed or were led to believe that these potent medications were in fact safe from a scientific standpoint. So, it was not the traditional "research-practice gap" that was to blame, but rather the misbelief, misinterpretation, and/or misapplication of the science. It is easy to see this in retrospect, but no one predicted it. The blithe acceptance and unquestioning citation by the medical community of a completely inadequate single publication regarding justification for safety were seized upon by the pharmaceutical industry. With seeming scientific assurance, the industry was able to deploy their significant public relations machinery to exert considerable weight and influence and subsequently increase prescribing of potent opioid medications to address pain. This effort was potentiated by a humane medical infrastructure push to address the debilitating human misery caused by acute and chronic pain. For a few years, while the focus lay on ensuring practitioners were assessing "the fifth vital sign," and prescribing accordingly, the waves on the surface appeared to be no cause for alarm. Yet, an invisible undertow was present and gathering momentum. This undercurrent would ultimately begin to sweep hundreds of thousands out to sea to drown beyond their depth, with even bigger waves of heroin and fentanyl to follow.

Going forward, one potential way to prevent this from happening again would be to create an annual independent scientific review and mandatory practitioner continuing education course on the science base for any new medications that have been adopted into health-care systems. This should be ordained as a national standard. In addition, continuing practice-based measurement and monitoring of the therapeutic effects of new medication implementation should be a part of routine care so that we move toward a system of "measurement-based practice" [37] that helps enhance quality and monitors safety in order to continually improve addiction health services. In addition, stricter regulations on the pharmaceutical industry to prohibit direct-to-consumer marketing and drastically curtail marketing to and conflicts of interest with physicians are desperately needed.

Another factor that needs to be addressed to prevent such a tragedy in the future is stigma and discrimination. The stigma and discrimination that pervades addiction meant a lethargic response to a rapidly growing public health crisis which, for almost any other health threat that might kill only a fraction of the number killed by the opioid overdose crisis, would have been met with greater alarm and more immediate and adequate appropriation from state and federal entities. A national response like those seen internationally to stem the threat from Ebola (Ebola virus disease [EVD]) or severe acute respiratory syndrome (SARS) would have curtailed this crisis. Instead, while the alarm bell was ringing there was a cultural and political deafness underwritten by prejudice against those suffering from addiction. This allowed an emerging crisis to grow and spread, change shape, and increase its toll until not a day passed without mention of the "opioid overdose crisis" on media channels of every political orientation and flavor. Only when it was realized that the overdose crisis kills equally across party lines, and the tears from dozens of bereaved mothers had moistened the 
benches of congress, did government begin to come together to act; but it was too little, too late, for hundreds of thousands of families. The pervasive impact of racism in our society's response to addiction crises and people who use drugs cannot be overemphasized. In past drug epidemics when the communities most deeply affected were black and Hispanic/Latino, the overwhelming response was a tough-on-crime approach that resulted in mass incarceration. The notion that the current crisis is in fact a public health issue was strongly influenced by the narrative of the innocent white victim, deserving of compassion rather than punishment. Addressing these pervasive stigmatizing attitudes through increasing education about the nature of opioid and other substance use disorders, shifting our language and terminology so that it more accurately reflects the neurological and medical nature of these conditions [38, 39], and increasing the personal testimony from the millions already in recovery [40] who are willing to speak out and demonstrate that recovering "addicts" are like everyone else, are ways likely to help reduce the prejudice, stigma, and discrimination that has retarded and undermined an adequate, rapid, response to the current crisis.

Finally, ideological stigma against proven effective medications also has meant too many have suffered and lost their lives when both might well have been avoided had providers, and politicians, understood the science on the effectiveness of these medications. Methadone, buprenorphine/naloxone, and extended-release naltrexone have among the strongest data of any intervention for any substance use disorder supporting their therapeutic benefits. Yet, people taking these highly effective medications remained misunderstood and, ironically, re-stigmatized as being "still using." Little attention was paid to acknowledging the clinical scientific data demonstrating that these medications dramatically reduce the risk of overdose death and enhance remission rates for OUD. Such prejudices against addiction treatment and medications in particular are driven by ignorance of the evidence base and misunderstanding of the nature of opioid addiction.

In sum, there are several hard lessons that might be learned from the rise and spread of the current opioid addiction and overdose death crisis. These pertain to recognizing the value of clinical science and the need to translate and understand it properly as well as respect it and the need to address stigma and misunderstanding both outside and inside the addiction field. Socially and culturally, attention to addiction has perhaps never been higher. The question for the future will be whether these grave and tragic lessons learned will be taken to heart and the structural changes made to prevent such crises from recurring in the future.

\section{References}

1. Moller LF, Matic S, van den Bergh BJ, Moloney K, Hayton P, Gatherer A. Acute drug-related mortality of people recently released from prisons. Public Health. 2010;124(11):637-9.

2. UNODC. United Nations Office on Drugs and Crime. World drug report 2017 [press release]. 2017.

3. Porter J, Jick H. Addiction rare in patients treated with narcotics. NEngl J Med. 1980;302(2):123.

4. Rosenstock I. Historical origins of the health belief model. Health Educ Monogr. 1974;2(4):328-35. 
5. 2015 National Survey on Drug Use and Health (NSDUH): methodological summary and definitions [Internet]. Substance Abuse and Mental Health Services Administration. 2016 [cited ]. Available from: https://www.samhsa.gov/data/sites/default/files/NSDUHMethodSummDefsHTML-2015/NSDUH-MethodSummDefsHTML-2015/NSDUHMethodSummDefs-2015.htm.

6. Cicero TJ, Ellis MS, Surratt HL, Kurtz SP. The changing face of heroin use in the United States: a retrospective analysis of the past 50 years. JAMA Psychiat. 2014;71(7):821-6.

7. Hedegaard H, Warner M, Minino AM. Drug overdose deaths in the United States, 1999-2016. NCHS Data Brief. 2017;(294):1-8.

8. Seth P, Scholl L, Rudd RA, Bacon S. Overdose deaths involving opioids, cocaine, and psychostimulants - United States, 2015-2016. MMWR Morb Mortal Wkly Rep. 2018;2018(67):349-58.

9. Barocas JA, White LF, Wang J, Walley AY, LaRochelle MR, Bernson D, et al. Estimated prevalence of opioid use disorder in Massachusetts, 2011-2015: a capture-recapture analysis. Am J Public Health. 2018;108(12):e1-7.

10. Dowell D, Haegerich TM, Chou R. CDC guideline for prescribing opioids for chronic pain-United States, 2016. JAMA. 2016;315(15):1624-45.

11. National Academies of Sciences E, and Medicine. Pain Management and the opioid epidemic. Balancing societal and individual benefits and risks of prescription opioid use. Washington, DC: The National Academies Press; 2017.

12. Mattick RP, Breen C, Kimber J, Davoli M. Buprenorphine maintenance versus placebo or methadone maintenance for opioid dependence. Cochrane Database Syst Rev. 2014;2:Cd002207.

13. Nielsen S, Larance B, Degenhardt L, Gowing L, Kehler C, Lintzeris N. Opioid agonist treatment for pharmaceutical opioid dependent people. Cochrane Database Syst Rev. 2016;(5):Cd011117.

14. Minozzi S, Amato L, Vecchi S, Davoli M, Kirchmayer U, Verster A. Oral naltrexone maintenance treatment for opioid dependence. Cochrane Database Syst Rev. 2011(4):Cd001333.

15. Lee JD, Nunes EV Jr, Novo P, Bachrach K, Bailey GL, Bhatt S, et al. Comparative effectiveness of extended-release naltrexone versus buprenorphine-naloxone for opioid relapse prevention (X:BOT): a multicentre, open-label, randomised controlled trial. Lancet. 2018;391(10118):309-18.

16. Jarvis BP, Holtyn AF, Subramaniam S, Tompkins DA, Oga EA, Bigelow G, et al. Extendedrelease injectable naltrexone (XR-NTX): a response to clinical issues raised by Brewer \& Streel. Addiction. 2019;114(1):189-90. https://doi.org/10.1111/add.14462. Epub 2018 Oct 30.

17. Kelly JF, White WL. Broadening the base of addiction mutual-help group organizations. J Groups Addict Recover. 2012;7(2-4):82-101.

18. Ling W, Hillhouse M, Ang A, Jenkins J, Fahey J. Comparison of behavioral treatment conditions in buprenorphine maintenance. Addiction. 2013;108(10):1788-98.

19. Wampold BE. The great psychotherapy debate: models, methods, and findings. Mahwah: L. Erlbaum Associates; 2001.

20. Longabaugh R, Magill M, Morgenstern J, Huebner R. Mechanisms of behavior change in treatment for alcohol and other drug use disorders. Addictions: a comprehensive guidebook. 2nd ed. New York: Oxford University Press; 2013. p. 572-96.

21. Dennis M, Godley SH, Diamond G, Tims FM, Babor T, Donaldson J, et al. The Cannabis Youth Treatment (CYT) Study: main findings from two randomized trials. J Subst Abus Treat. 2004;27(3):197-213.

22. Gossop M, Marsden J, Stewart D. Remission of psychiatric symptoms among drug misusers after drug dependence treatment. J Nerv Ment Dis. 2006;194(11):826-32.

23. Monico LB, Gryczynski J, Mitchell SG, Schwartz RP, O'Grady KE, Jaffe JH. Buprenorphine treatment and 12-step meeting attendance: conflicts, compatibilities, and patient outcomes. J Subst Abus Treat. 2015;57:89-95.

24. Brown RT. Systematic review of the impact of adult drug-treatment courts. Transl Res. 2010;155(6):263-74. 
25. Wittouck C, Dekkers A, De Ruyver B, Vanderplasschen W, Vander Laenen F. The impact of drug treatment courts on recovery: a systematic review. ScientificWorldJournal. 2013;2013:493679.

26. Kelly JF, Finney JW, Moos R. Substance use disorder patients who are mandated to treatment: characteristics, treatment process, and 1- and 5-year outcomes. J Subst Abus Treat. 2005;28(3):213-23.

27. Dennis ML, Scott CK, Funk R, Foss MA. The duration and correlates of addiction and treatment careers. J Subst Abus Treat. 2005;28(Suppl 1):S51-62.

28. Dennis ML, Foss MA, Scott CK. An eight-year perspective on the relationship between the duration of abstinence and other aspects of recovery. Eval Rev. 2007;31(6):585-612.

29. Sordo L, Barrio G, Bravo MJ, Indave BI, Degenhardt L, Wiessing L, et al. Mortality risk during and after opioid substitution treatment: systematic review and meta-analysis of cohort studies. BMJ. 2017;357:j1550.

30. Hser YI, Huang D, Saxon AJ, Woody G, Moskowitz AL, Matthews AG, et al. Distinctive trajectories of opioid use over an extended follow-up of patients in a multisite trial on Buprenorphine + Naloxone and Methadone. J Addict Med. 2017;11(1):63-9.

31. Strang J, Groshkova T, Uchtenhagen A, van den Brink W, Haasen C, Schechter MT, et al. Heroin on trial: systematic review and meta-analysis of randomised trials of diamorphine-prescribing as treatment for refractory heroin addiction dagger. Br J Psychiatry. 2015;207(1):5-14.

32. Stevens EB, Jason LA, Ferrari JR. Measurement performance of the sense of community index in substance abuse recovery communal housing. Aust Community Psychol. 2011;23(2):135-47.

33. Fallah-Sohy N, Vilsaint CL, Cristello JV, O'Connor CL, Jason LA, Stout RL, et al. Characterization of addiction recovery community centers in the Northeastern United States. Alcohol Clin Exp Res. 2016;40(S1):683.

34. Abdul-Quader AS, Feelemyer J, Modi S, Stein ES, Briceno A, Semaan S, et al. Effectiveness of structural-level needle/syringe programs to reduce HCV and HIV infection among people who inject drugs: a systematic review. AIDS Behav. 2013;17(9):2878-92.

35. Aspinall EJ, Nambiar D, Goldberg DJ, Hickman M, Weir A, Van Velzen E, et al. Are needle and syringe programmes associated with a reduction in HIV transmission among people who inject drugs: a systematic review and meta-analysis. Int J Epidemiol. 2014;43(1):235-48.

36. Potier C, Laprevote V, Dubois-Arber F, Cottencin O, Rolland B. Supervised injection services: what has been demonstrated? A systematic literature review. Drug Alcohol Depend. 2014;145:48-68.

37. Kelly JF, Mee-Lee D. Quality, accountability, and effectiveness in addiction treatment: the measurement-based practice model. In: Danovitch I, Mooney L, editors. The assessment and treatment of addiction: best practices and new frontiers. 1st ed. St. Louis: Elsevier; 2018.

38. Kelly JF, Saitz R, Wakeman SE. Language, substance use disorders, and policy: the need to reach consensus on an "addiction-ary". Alcohol Treat Q. 2016;34(1):116-23.

39. Kelly JF, Dow S, Westerhoff C. Does our choice of substance-related terminology influence perceptions of treatment need? An empirical investigation with two commonly used terms. J Drug Issues. 2010;40(4):805-18.

40. Kelly JF, Bergman BG, Hoeppner BB, Vilsaint CL, White WL. Prevalence and pathways of recovery from drug and alcohol problems in the United States population: implications for practice, research, and policy. Drug Alcohol Depend. 2017;181:162-9. 